\title{
Diseño de un dinamómetro para la evaluación objetiva de los músculos motores del brazo
}

\author{
Lucas Bisio ${ }^{1}$, Gastón Gutierrez ${ }^{1}$, Aníbal Marquez ${ }^{1}$, Rosanna De Falco², \\ Sandra Porro ${ }^{2}$ y Adrián P. Cisilino ${ }^{1}$
}

\begin{abstract}
Resumen
Las pruebas de fuerza muscular proporcionan información fundamental para el diagnóstico diferencial, pronóstico y tratamiento de los trastornos neuromusculares y músculo-esqueléticos en el miembro superior. Las evaluaciones utilizadas en la actualidad se basan en métodos manuales que utilizan escalas de registro ambiguas que dependen más de la capacidad muscular del examinador que la del evaluado.

Con la finalidad de disponer de una metodología objetiva para valoración de la capacidad funcional del miembro superior se presenta en este trabajo el diseño de un dinamómetro para la medición instrumentada de las fuerzas de los músculos motores primarios del brazo. El diseño propuesto resulta en una aparatología discreta, portátil y con adquisición computarizada de los datos. Al mismo tiempo, posibilita el necesario contacto entre el examinador y el evaluado para realizar las maniobras de fijación, estabilización y verificación de tonicidad muscular.
\end{abstract}

Palabras clave: • Miembro superior • Fuerzas musculares • Dinamómetro de banco

\section{Design of a dynamometer for the objective evaluation of the motive muscles of the arm}

\begin{abstract} examiner than that of the person under evaluation.

It is presented in this work the design of a dynamometer for the evaluation of the forces exerted by the motive muscles of the arm. The proposed device aims to the development of an objective methodology for the assessment of the functional capacity of the upper limb. The design consists in a simple portable device with contact between the examiner and the evaluated person during the maneuvers of fixation, stabilization and muscle tone verification.

Keywords: • Upper limb • Muscular forces • Dynamometer

${ }^{1}$ División Soldadura y Fractomecánica INTEMA -, Departamento de Ingeniería Mecánica Facultad de Ingeniería, Universidad Nacional de Mar del Plata.

Av. Juan B. Justo 4302 (7600) Mar del Plata, Argentina.Email: cisilino@fi.mdp.edu.ar

${ }^{2}$ Departamento de Terapia Ocupacional.

Facultad de Ciencias de la Salud y Servicio Social,Universidad Nacional de Mar del Plata

Funes3350 Nivel +4 (7600) Mar del Plata, Argentina.Email: centrodemano@yahoo.com
\end{abstract}

Muscle force evaluation provides fundamental information for the diagnosis, prediction and treatment of neuro-muscular and muscle-skeletal disorders in the upper limb. Current evaluation techniques are based on manual methods with ambiguous valuation scales that depend more of the muscular capacity of the an automated data acquisition system. At the same time, it allows the necessary 


\section{Introducción}

Dada la importancia de la vinculación de los miembros superiores con el medio que los circunda estos cumplen quizás el papel más importante en nuestra actividad diaria, por lo que las afecciones inflamatorias o degenerativas son muy comunes, en especial en el complejo articular del hombro. Las molestias iniciales en el miembro superior son generalmente ignoradas o menospreciadas, hasta que el dolor se agudiza profundamente provocando una pronunciada disminución de su movilidad. Un alto porcentaje de estas lesiones son provocadas en actividades físicas relacionadas con el deporte, pero aquellas que tienen que ver con la actividad laboral traen consigo una serie de inconvenientes que van más allá de la misma lesión y que tienen que ver con el aspecto individual y social del trabajador. Una lesión produce una ausencia parcial o total en el puesto de trabajo, repercute psíquicamente en el individuo que requiere de tratamiento medico, con su inevitable costo asociado, y por último y más importante, puede resultar en una discapacidad temporal o definitiva que le impida al paciente retomar sus tareas con el nivel de eficiencia anterior a la lesión. Es así que la disponibilidad de equipamiento, técnicas y recursos humanos con la capacidad de diagnosticar y brindar soluciones a las dolencias del miembro superior resultan no solo en beneficios económicos contribuyendo al incremento de los índices de integración laboral y la estabilidad en el empleo, sino que también aporta a la mejora de la calidad de vida de los pacientes.

Es en este contexto que este trabajo presenta el diseño de un equipo de banco para la medición de las fuerzas musculares del miembro superior con el objetivo de disponer una metodología objetiva que sirva como complemento para el diagnóstico, evaluación, control de evolución, planificación $\mathbf{y}$ herramienta de investigación en los tratamientos de rehabilitación en pacientes con alteraciones anátomo-funcionales.

\section{Materiales y Métodos}

\section{El examen de evaluación de fuerza muscular}

La fuerza muscular es la expresión de la tensión muscular transmitida al hueso a través del tendón. Como fue ya antes mencionado, el método de evaluación de fuerza muscular más difundido es el de valoración manual. Este método utiliza una escala de registro basada en la capacidad de un músculo en realizar el desplazamiento de las palancas en relación a la gravedad, como se indica en la Tabla 1 [1].

\begin{tabular}{|c|l|}
\hline Registro & \multicolumn{1}{c|}{ Capacidad del músculo } \\
\hline M0 & Parálisis total \\
\hline M1 & Contracción sin desplazamiento de palancas \\
\hline M2 & Desplazamiento de palancas sin acción de la gravedad \\
\hline M3 & Desplazamiento de palancas contra la acción de la gravedad \\
\hline M4 & Desplazamiento de palancas contra resistencia moderada \\
\hline M5 & Desplazamiento de palancas contra resistencia máxima \\
\hline
\end{tabular}

Tabla 1: Escala de registro de capacidad muscular

El método de valoración manual presenta una serie de limitaciones. Por un lado la acción de la gravedad sobre el desplazamiento de palancas es un factor que interviene solo en el $60 \%$ de los músculos de las extremidades, por lo que la escala de la Tabla 1 no es apta para la 
evaluación de todos los músculos del miembro superior. A su vez, los conceptos de resistencia moderada y resistencia máxima son ambiguos y dependen más de la capacidad muscular del examinador que la del evaluado [2]. Kendall et al [1] refiriéndose a las pruebas musculares de valoración manual observan que "los resultados solo son útiles cuando se registran con exactitud, ya que una prueba inadecuada solo puede conducir a confusión" y además agregan que "las mismas representan un procedimiento que depende del conocimiento, destreza y experiencia del examinador".

\section{Antecedentes y requisitos de diseño}

En los últimos años se han desarrollado dispositivos para medir fuerzas musculares del miembro superior que consisten en sensores que deben ser sostenidos por el examinador (ver por ejemplo el Lafayette Manual Muscle Test System [3]). Aunque estos dispositivos presentan una mejora sobre el método manual al permitir cuantificar el valor de las fuerzas, conservan la limitación de que la fuerza del examinador es la que gradúa la resistencia opuesta al músculo evaluado. Otros equipamientos más sofisticados son por ejemplo el ULTMS (Upper Limb Tone Measurement System) desarrollado por la Universidad de Johns Hopkins [4], dispositivo robótico automatizado para cuantificar el tono de los grupos musculares flexores y extensores de la muñeca, y el comercializado por la empresa Quantitative Muscle Assessment [5] que combina sensores de fuerza junto con análisis de ultrasonido para la identificación del músculo. Debido a su elevado precio, estos dispositivos son de difícil acceso y no se encuentran disponibles en nuestro país.

Se propone en este trabajo diseñar un dispositivo sencillo y portátil que sirva para valorar en forma objetiva las fuerzas musculares del miembro superior. El diseño debe contemplar los siguientes aspectos:

- Contar con la capacidad para explorar en la forma individual los músculos motores primarios del brazo: deltoides anterior, deltoides medio, deltoides posterior, bíceps y tríceps. Si bien existen otros grupos musculares en el brazo, se considera a estos cinco como los de principal importancia ya que brindan el mayor caudal de información sobre el estado del paciente.

- El paciente debe estar en una posición que asegure que las partes o regiones del cuerpo que no son objeto del examen se mantengan en la forma más firme y estable posible. La posición sentada es la más adecuada para el examen del miembro superior con los tobillos en $90^{\circ}$ de flexión dorsal, rodilla en flexión de $90^{\circ}$, cadera en flexión de $90^{\circ}$ y apoyo lumbar sobre el respaldo. Altura del respaldo: hasta columna dorsal. La altura del asiento deberá ser regulable para adaptarse a las dimensiones de cada paciente.

- Debe asegurarse que la región proximal del segmento explorado se encuentre estabilizado. La calidad de la fijación depende en gran medida de la silla de exploración. El examen no será adecuado si el paciente se apoya sobre un lecho blando que se hunde cuando se realiza la acción muscular. La fijación de la zona proximal de la extremidad podrá ser realizada por un estático del dispositivo explorador o por el examinador.

- El dispositivo debe ser discreto, es decir no debe intimidar al paciente. Al mismo tiempo debe permitir el contacto entre el examinador y el evaluado para realizar las maniobras de fijación, estabilización y verificación de tonicidad muscular.

- Debe contar con un sistema para el registro de datos. 


\section{Resultados}

Las Figuras 1 y 2 presentan vistas del diseño propuesto. En la Figura 1 se indica la posición del paciente y la geometría del dispositivo plegado, mientras que en la Figura 2 se indican sus movimientos principales. Se trata de un dispositivo totalmente pasivo que es en esencia una balanza electrónica orientable con la posibilidad de registrar la posición de los sensores para tener repetitividad en las mediciones.

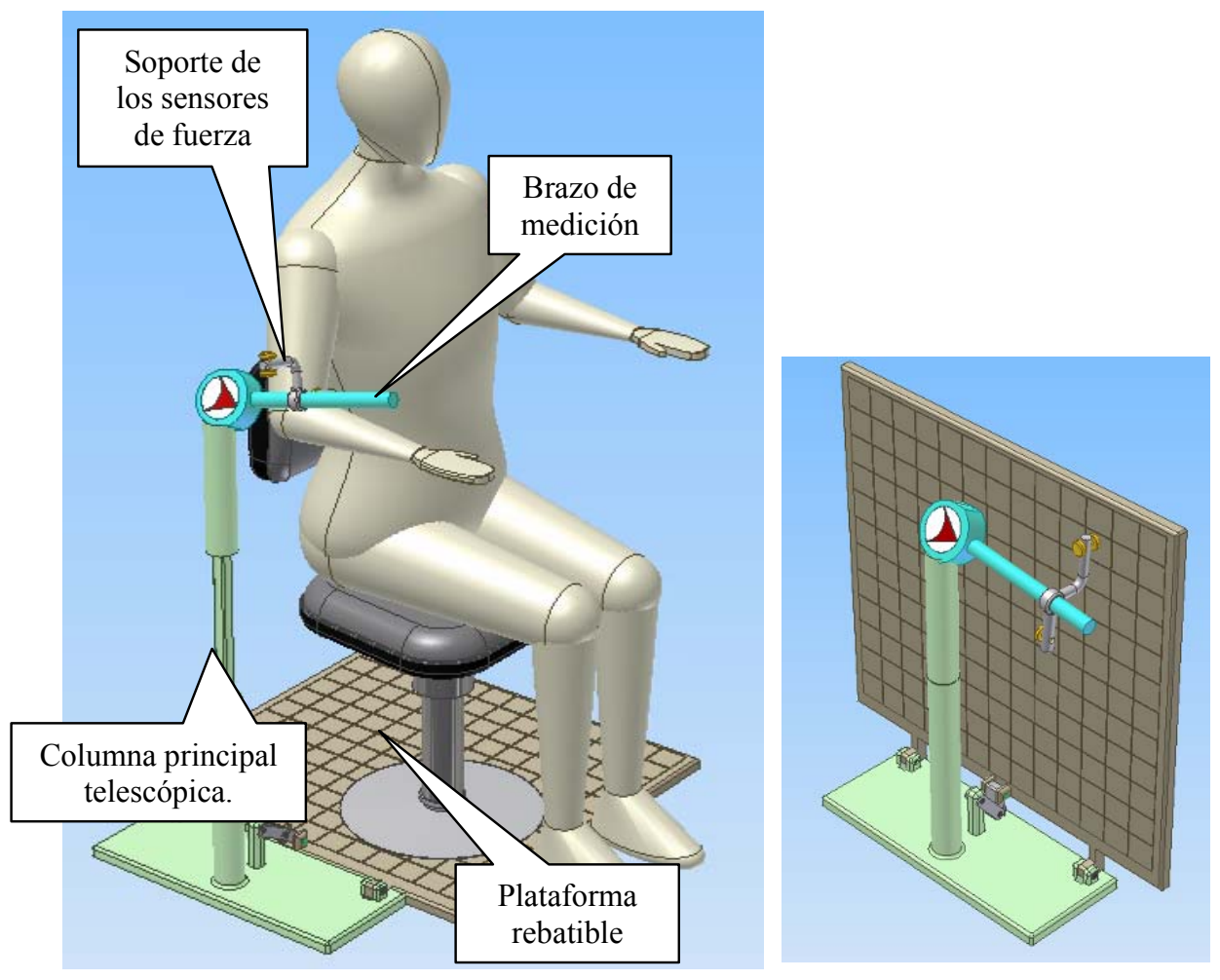

Figura 1: Modelo tridimensional del dispositivo de medición en referencia la posición del paciente. A la derecha posición plegada.

El dispositivo fue diseñado para medir las fuerzas de los cinco músculos motores principales del brazo según el procedimiento que se resume en la Tabla 2. Este procedimiento es una adaptación del utilizado por el método de valoración manual (ver Kendall et al [1]) para la utilización del dispositivo propuesto. Es interesante notar que para la secuencia de posiciones de la Tabla 2 el brazo del paciente describe aproximadamente un cono con vértice en la articulación del hombro. Sobre la base de esta observación se estudió la cinemática del dispositivo de manera tal que sea posible, para todas en las posturas indicadas, ubicar los sensores de fuerza en posiciones conjugadas a las del brazo del paciente.

El dispositivo consta de una columna vertical principal que sostiene y posiciona en altura al brazo de medición. La columna tiene un mecanismo telescópico neumático de longitud regulable y bloqueo mediante una válvula hidráulica, similar a las utilizadas en el pie de los muebles de oficina. Esto permite su posicionamiento a diferentes alturas compensando el peso de la estructura superior.

El brazo de medición sirve de guía para el soporte de los sensores. El brazo de medición gira alrededor de un eje solidario a la columna e inclinado $30^{\circ}$ grados respecto de la horizontal. El 
giro del brazo de medición puede bloquearse en posiciones predeterminadas mediante una traba ubicada en la articulación del brazo de medición y la columna.

El soporte de los sensores puede deslizar y girar sobre el brazo de medición. Su posición se fija mediante un mecanismo de fricción contra el brazo de medición. El posicionamiento de los sensores a lo largo del brazo de medición y con el control de altura de la columna permiten adaptar el dispositivo a la contextura de cada paciente. El desplazamiento y el giro de los sensores y la altura de la columna pueden ser medidos mediante escalas graduadas y registrados junto con los datos de fuerza para asegurar la repetitividad de la medición.

La fuerza ejercida por el paciente es medida por los sensores de fuerza y registrada en forma continua a través de un sistema de adquisición y acondicionamiento de señal conectado a una PC a través de una interfase USB. Los sensores están dispuestos en alojamientos en el soporte mediante trabas magnéticas o velcro. Se prevé la utilización de tres sensores, uno para la medición de bíceps y tríceps, uno para deltoides medio y otro para anterior y posterior. Eventualmente se pondrá utilizar un solo sensor, alternado su colocación en los diferentes alojamientos.

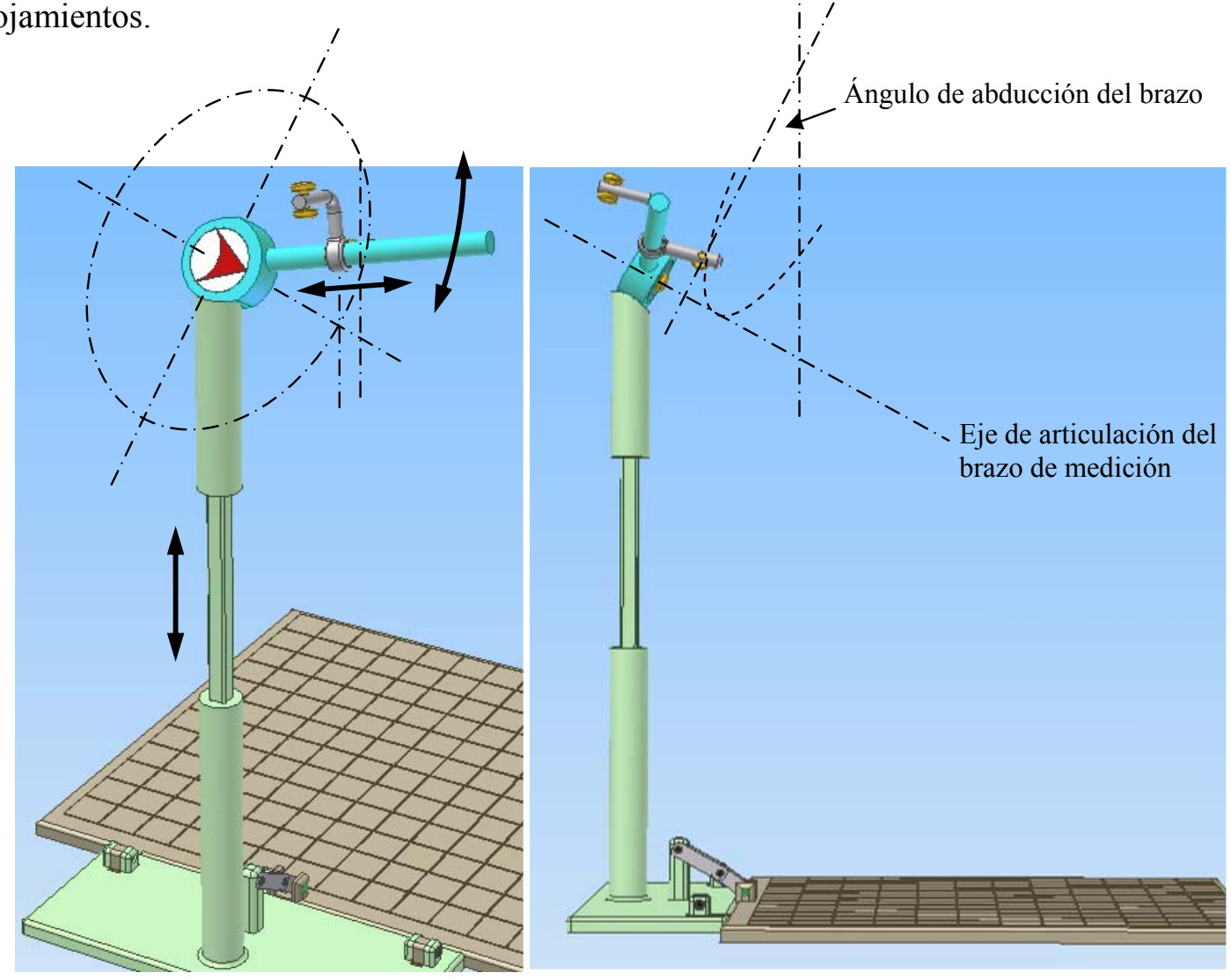

Figura 2: Descripción de los movimientos para el posicionamiento del dispositivo de medición de fuerza.

Los datos adquiridos son procesados por programa desarrollado en el entorno de software LabVIEW. El programa contará con una interfase amigable para el usuario para la toma de datos y el archivo de los parámetros de la prueba (altura del brazo, posición de los sensores, etc.).

A partir de la base del soporte de la columna principal se despliega una plataforma rebatible sobre la que se coloca la silla. El peso propio del paciente sobre la plataforma brinda estabilidad al sistema. Dependiendo de la posición de la silla sobre la plataforma el 
dispositivo sirve para el examen de ambos brazos. Esta disposición se adoptó con el objetivo de reducir el espacio necesario para el almacenamiento del dispositivo cuando está fuera de uso y facilitar su transporte en el caso de prácticas fuera del consultorio (domiciliarias o en hospitales). Se estima que el peso máximo del dispositivo será de $15 \mathrm{Kg}$ y sus dimensiones plegado $0,6 \mathrm{~m} \times 0,6 \mathrm{~m} \times 0,3 \mathrm{~m}$. Desde el punto de vista estructural todos los elementos están dimensionados para presentar la necesaria rigidez frente a las máximas fuerzas que pueda ejercer un paciente, así como para el rango máximo de medición $(100 \mathrm{Kg})$.

\begin{tabular}{|c|c|}
\hline $\begin{array}{l}\text { Deltoides } \\
\text { anterior }\end{array}$ & $\begin{array}{l}\text { Acción primaria: Flexor de la articulación gleno-humeral. } \\
\text { Posición: Hombro en leve abducción y rotación interna de } 30^{\circ} \text {. } \\
\text { Zona de fijación: Escápula. } \\
\text { Dirección de movimiento: Plano sagital, dirección anterior. } \\
\text { Orden verbal: "Llevar el brazo hacia adelante". } \\
\text { Punto de aplicación: Proximal a la articulación del codo. }\end{array}$ \\
\hline $\begin{array}{l}\text { Deltoides } \\
\text { medio }\end{array}$ & $\begin{array}{l}\text { Acción primaria: Abducción de la articulación gleno-humeral. } \\
\text { Posición: Hombro en abducción de } 30^{\circ} \text {. Rotación neutra. } \\
\text { Zona de fijación: Escápula. } \\
\text { Dirección de movimiento: Plano frontal, dirección externa. } \\
\text { Orden verbal: "Llevar el brazo hacia el costado". } \\
\text { Punto de aplicación: Proximal a la articulación del codo, zona lateral } \\
\text { externa de brazo, por encima de epicóndilo. }\end{array}$ \\
\hline $\begin{array}{l}\text { Deltoides } \\
\text { posterior }\end{array}$ & $\begin{array}{l}\text { Acción primaria: Extensor de la articulación gleno-humeral. } \\
\text { Posición: Hombro en leve abducción y rotación neutra. } \\
\text { Zona de fijación: Escápula. } \\
\text { Dirección de movimiento: Plano sagital, dirección posterior. } \\
\text { Orden verbal: "Llevar el brazo hacia atrás". } \\
\text { Punto de aplicación: Proximal a la articulación del codo, zona posterior del } \\
\text { brazo, por encima del olecranon. }\end{array}$ \\
\hline Bíceps & $\begin{array}{l}\text { Acción primaria: Flexor de la articulación del codo. } \\
\text { Posición: Hombro en posición de relax. Codo en } 90^{\circ} \text { de flexión. Antebrazo } \\
\text { en supinación. } \\
\text { Zona de fijación: Brazo. } \\
\text { Dirección de movimiento: Plano sagital, dirección anterior. } \\
\text { Orden verbal: "Flexionar el codo". } \\
\text { Punto de aplicación: Proximal a la articulación de muñeca, cara anterior del } \\
\text { antebrazo. }\end{array}$ \\
\hline Tríceps & $\begin{array}{l}\text { Acción primaria: Extensor de la articulación del codo. } \\
\text { Posición: Hombro en adducción completa y rotación neutra. Codo en } \\
\text { flexión de } 90^{\circ} \text {. Antebrazo en pronación. } \\
\text { Zona de fijación: Brazo. } \\
\text { Dirección de movimiento: Plano sagital, dirección posterior. } \\
\text { Orden verbal: "Llevar el brazo hacia abajo". } \\
\text { Punto de aplicación: Proximal a la articulación de la muñeca, cara anterior } \\
\text { del antebrazo. }\end{array}$ \\
\hline
\end{tabular}

Tabla 2: Detalle de condiciones para el examen de los músculos motores principales del brazo. 
La Figura 4 presenta a modo de ejemplo la medición del deltoides anterior y medio. Se presenta en cada caso una fotografía tomada de Kendall et al [1] donde se ilustra la posición del paciente, la fijación y la evaluación de la fuerza muscular por parte del evaluador. Las flechas blancas indican la acción del músculo, mientras que las flechas rojas corresponden a la reacción medida en el punto de resistencia. Por su parte las figuras que ilustran el dispositivo indican la posición del brazo de medición y la posición del sensor de carga. Se destaca que el diseño esbelto del dispositivo de medición permite al evaluador tener un acceso cómodo al paciente y de esta forma realizar las maniobras de fijación, estabilización y verificación de tonicidad muscular.

\section{Conclusiones}

Se presentó el diseño de un dispositivo para la medición cuantitativa y objetiva de las fuerzas de los músculos motores principales del miembro superior. La metodología propuesta involucra la adquisición y registro de datos mediante PC y deja a cargo del evaluador las maniobras de fijación, estabilización y verificación del tono muscular durante la medición.

El peso y las dimensiones del dispositivo propuesto lo hacen apto para la práctica en consultorios con espacio limitado y transportable para prácticas hospitalaria y domiciliaria.

El dispositivo presentado se encuentra actualmente en etapa de construcción. Finalizada la misma, la siguiente etapa del proyecto consistirá en la calibración y adaptación de la metodología de medición de fuerzas. Se realizarán estudios estadísticos de medición de fuerza que servirán para elaborar estándares y recomendaciones para la utilización del dispositivo.

Se presentaran como uno de los objetivos de investigación la cuantificación de la subjetividad de la escala de valoración manual (ver Tabla 1) mediante la comparación de mediciones realizadas por distintos examinadores sobre un único grupo de pacientes. Para esto se compararán y correlacionarán los resultados de mediciones obtenidas utilizando la escala de valoración manual y resultados obtenidos de mediciones realizadas mediante la utilización de la herramienta propuesta en este trabajo.

En etapas más avanzadas del proyecto se planea utilizar el dispositivo para registrar la evolución de las fuerzas musculares durante tratamientos de rehabilitación aplicado en diferentes patologías del miembro superior Estos datos complementarán el historial de la evolución del paciente y servirán para evaluar la efectividad de los protocolos utilizados durante el abordaje de patologías tales como lesiones tendinosas, parálisis periféricas, patologías degenerativas y fracturas.

\section{Discusión}

\section{$\underline{\text { Respuestas a los revisores }}^{1}$}

1. Como bien señalan los revisores el artículo trata sobre el diseño de un instrumento de medición, el que actualmente se encuentra en la etapa de construcción. Es por esto que no se reportan resultados sobre pruebas o la calibración del instrumento. Con el objetivo de evitar malas interpretaciones por parte del lector, se ha enfatizado este punto en la sección Conclusiones del manuscrito.

\footnotetext{
${ }^{1}$ Nota de la edición: sea juntan estos argumentos para validar la publicación del articulo. Durante las revisiones se objetó el trabajo por no referir su utilidad desde su aplicación consolidada. Mas tarde y frente a las explicaciones dadas me acepta como un proyecto en desarrollo.
} 
2. Se enuncia en las Conclusiones información sobre las pruebas, calibraciones y aplicaciones que se planean para el instrumento.

3. Uno de los revisores observa la falta de principios científicos sobre los que se fundamenta el diseño del instrumento. En este sentido señalamos que el diseño está fundamentado sobre el método de evaluación manual. Es por esto que el diseño del instrumento deja a cargo del evaluador las maniobras de fijación, estabilización y verificación del tono muscular durante la medición. El instrumento asiste al examinador con la adquisición y registro de datos de fuerzas para reducir la subjetividad de los resultados de la evaluación. La metodología propuesta mantiene los procedimientos y técnicas actualmente utilizado y aceptado (Figura 3).

4. Los autores no ignoramos que la valoración de la capacidad funcional del miembro superior se realiza a través de múltiples pruebas y evaluaciones que exceden al examen de la fuerza muscular. El instrumento propuesto no pretende reemplazar a los exámenes a los que se refiere el revisor, sino que los asiste y complementa.

\section{Agradecimientos}

Este trabajo ha sido financiado por el proyecto PICT 12-14114 "Biomecánica del Miembro Superior" de la Agencia Nacional de Promoción Científica de la República Argentina y Tecnológica y el Proyecto ING15G/168 de la Universidad Nacional de Mar del Plata. 

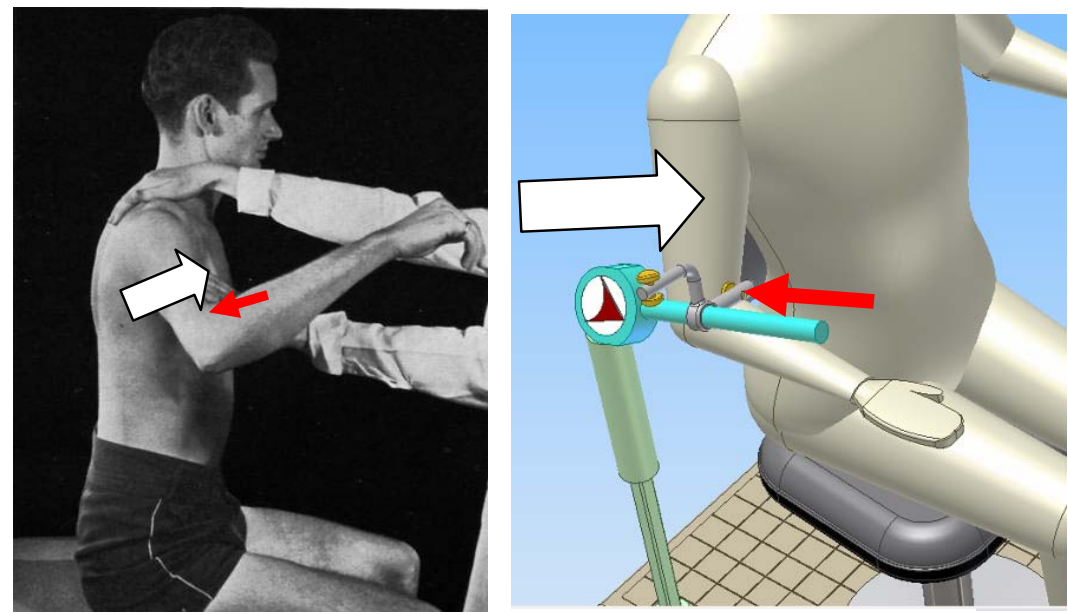

(a) Deltoides anterior
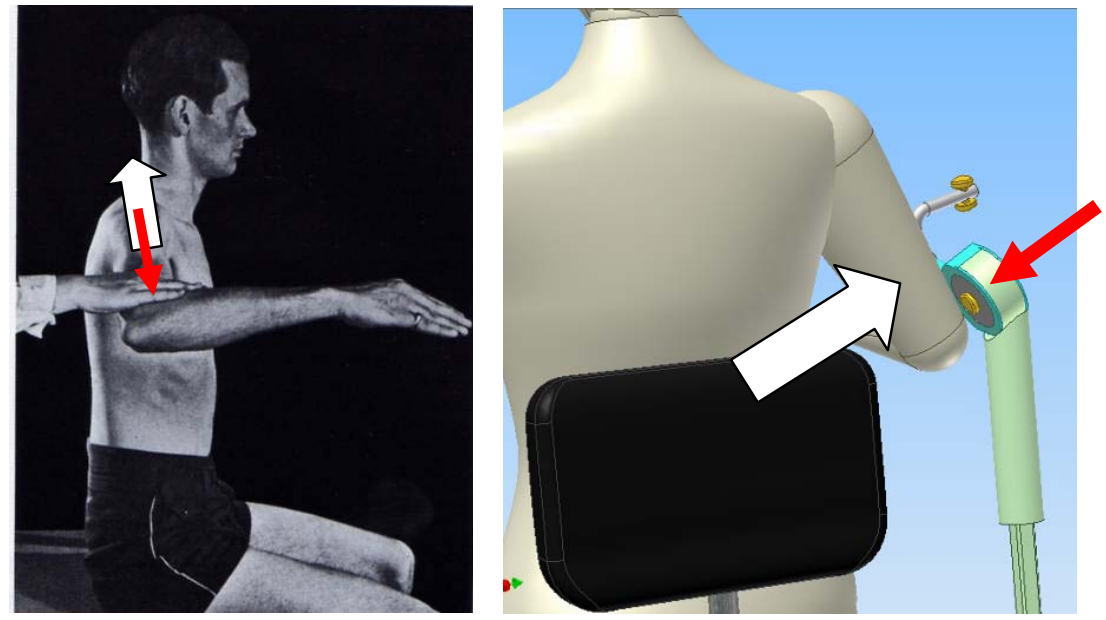

(b) Deltoides medio

Figura 3: Posiciones de medición para los músculos deltoides (a) anterior y (b) medio. 


\section{Referencias}

1. Kendall F.P., Kendall E. y Mc Creary E. "Músculos, Pruebas y Funciones". Ed. Jims, Barcelona (1985). Cap 1, pp. 1-17; Cap. 4 pp. 59-128.

2. Hunther J.M., MacKin E.J. y Dallahan A.D. "Rehabilitation of the Hand: Surgery and Therapy", Ed. Mosby (1995). Part IV Cap. 11, pp. 153-183; Cap. 12 pp. 185 -213.

3. Lafayette Manual Muscle Test System, Lafayette Instrument Company, Lafayette, IN 47903, USA. Disponible en http://www.lafayetteevaluation.com (revisado en octubre de 2008)

4. Department of Physical Medicine \& Rehabilitation, Universidad Johns Hopkins, Baltimore, Maryland, USA. Disponible en

http://www.hopkinsmedicine.org/Rehab/research/biomech.html (revisado en octubre de 2008) 5. Quantitative Muscle Assessment, Aeverl Medical, Gainesville, Georgia, USA. Disponible en http://www.qmasystem.com (revisado en octubre de 2008) 